\title{
Exploring the Orthogonal Relationship between Controlled and Automated Processes in Skilled Action
}

\author{
John Toner $^{1}$ (D) Aidan Moran ${ }^{2}$ \\ Published online: 22 August 2020 \\ (C) The Author(s) 2020
}

\begin{abstract}
Traditional models of skill learning posit that skilled action unfolds in an automatic manner and that control will prove deleterious to movement and performance proficiency. These perspectives assume that automated processes are characterised by low levels of control and vice versa. By contrast, a number of authors have recently put forward hybrid theories of skilled action which have sought to capture the close integration between fine-grained automatic motor routines and intentional states. Drawing heavily on the work of Bebko et al. (2005) and Christensen et al. (2016), we argue that controlled and automated processes must operate in parallel if skilled performers are to address the wide range of challenges that they are faced with in training and competition. More specifically, we show how skilled performers use controlled processes to update and improve motor execution in training contexts and to stabilise performance under pressurised conditions.
\end{abstract}

Keywords Control · Automaticity $\cdot$ Continuous improvement · Expertise $\cdot$ Skilled action

\section{Introduction}

Skilled action in sport is widely believed to be facilitated by an absence of conscious attention to the mechanics of one's movements during skill execution (see Masters and Maxwell, 2008, for a review). Anecdotal evidence to support this idea abounds in sport.

John Toner

john.toner@hull.ac.uk

Aidan Moran

aidan.moran@ucd.ie

1 Department of Sport, Health and Exercise Sciences, University of Hull, Cottingham Road, Hull HU6 7RX, UK

2 School of Psychology, University College Dublin, Belfield, Dublin 4, Ireland 
For example, when the triple-major champion golfer Padraig Harrington won the 2007 open Championship in Carnoustie after a play-off against Sergio Garcia, he described his thoughts as he prepared for his final putt: 'no conscious effort whatsoever went into that putt. There were no thoughts about 'this is for the Open'... I stroked it in' (cited in Jones, 2007, p. 12). Supporting this idea, evidence from the experimental psychological literature would suggest that engaging in any form of conscious control will hamper athletic performance. To illustrate, performance pressure may lead athletes to become increasingly conscious of their movement and suffer a concomitant breakdown of automated skills (Beilock et al., 2002; Masters \& Maxwell, 2008) - a phenomenon popularly known as 'paralysis-by-analysis' (Beilock, 2010). More generally, a considerable body of experimental evidence highlights the debilitating effect of 'conscious processing' (or paying attention to one's action during motor skill execution) on skilled athletes' movement and performance (e.g., Beilock and Carr, 2001).

These findings support the predictions made by a number of highly influential models of skill acquisition which place controlled and automated processing at opposite ends of a single continuum (e.g., Shiffrin and Schneider, 1977). According to this view, processing becomes less deliberate and effortful (i.e., controlled) as a skill becomes more automatized. However, we argue that this perspective is unable to account for the wide range of empirical evidence which indicates that performance continues to be mediated by controlled processes even after one attains expertise. We seek to address this misunderstanding by presenting a model which explains how controlled and automated processes may emerge concurrently and how they must operate in a synergistic fashion if experts are to overcome the challenges they face in seeking to maintain performance proficiency over long timescales. We start by pointing to some of the weaknesses associated with linear or serial models of skill learning and the experimental paradigms used by studies they have spawned. Following this, we introduce a model of skilled action which explains how controlled and automatic processes operate in an orthogonal manner. Next, we elucidate what is meant by the term "controlled processes" before considering how expert performers will often revert to controlled processing when confronted by "bodily crises" and as part of ongoing attempts to maintain or update their bodily capacities. We then consider the role controlled processes play during peak performance states and detail some of the strategies athletes might use to induce clutch performances. We conclude by briefly discussing the role metacognition plays in allowing expert performers to identify and apply situationally appropriate modes of control.

At first glance, the body of empirical evidence which has explored the role consciousness plays in expert action would seem to suggest that conscious control will inevitably prove to be a hazardous activity for athletes to engage in during performance. But does this evidence tell the whole story? To answer this question, we need to delve deeper into the issues involved. One of the oldest distinctions in cognitive psychology is that between mental processes that require conscious control and those that do not. For example, William James (1892) distinguished between 'willed' actions (which involve a conscious component in the form of a 'fiat, mandate or expressed consent', $p$. 335) and 'ideo-motor' actions (where the person is 'aware of nothing between the conceptions and the execution', p. 335). This distinction surfaced again in cognitive psychology in the 1970s when researchers differentiated between 'controlled' processes (e.g., reading for comprehension), which are typically portrayed as being effortful, 
conscious, slow and error-prone and 'automatic' processes (e.g., writing one's name), which are regarded as being unintentional, effortless, involuntary, unconscious and fast (e.g., see Shiffrin and Schneider, 1977). In an effort to account for certain subsequent empirical anomalies (e.g., the fact that some automatic processes are amenable to consciousness and can be controlled intentionally whereas others are not; Sternberg and Sternberg, 2009), 'dual process' views of attention (e.g., Evans and Stanovich, 2013) have given way to a more fine-grained analysis of this construct. For example, a number of researchers have proposed hybrid theories of skilled action which have sought to capture the synthesis between cognitive and automatic processes (e.g., Mylopoulos and Pacherie, 2017).

And yet, despite the emergence of these latter perspectives, dual process views of skill learning (which place cognitive processes on a continuum ranging from fully controlled to fully automatic) continue to hold sway in the skill acquisition/sport psychology literature (e.g., Furley et al., 2015). Before considering the utility of hybrid theories, however, we should provide a brief overview of the dual process view and consider how this perspective has shaped current conceptualisations of skilled action. Dual process views typically portray learning as involving a gradual reduction of cognitive resources as one acquires an increasing level of control over motor execution. Take, for example, Fitts and Posner's (1967) highly influential model of skill acquisition which postulates that learning occurs in three linear stages. In the initial cognitive stage, agents draw on explicit rules to consciously guide task performance. This will inevitably require them to experiment with different strategies to see which brings them closer to a movement goal (Wulf, 2007). The associative stage is characterised by increasingly consistent and economical movement patterns. In a final autonomous stage, movement production is fluent, effortless, immune to dual task interference, and requires little cognitive control. Similar to a number of other influential models of skill learning (e.g., Anderson's, 1982, Adaptive Control of Thought Theory), Fitts and Posner theorise that controlled and automatic processes reside at opposite ends of a single continuum. According to this view, low automaticity is associated with high levels of control and vice versa.

Another feature common to dual process views is the belief that automaticity is a defining feature of optimal performance while control is associated with the degradation of motor execution (Masters and Maxwell, 2008). Advocates of this perspective often present the phenomenon of "expert induced amnesia" (when asked to recall how they have performed a task experts provide impoverished accounts containing little recollection of the episodic 'rules' that guided their action; see Beilock et al. 2002) as prima facie evidence that skilled actions are governed by automatic processes. In a number of experimental studies, skilled performers have reported few explicit episodic rules relating to the execution of a highly practiced motor skill (e.g., Beilock et al. 2002). Unfortunately, the experimental paradigm adopted by many of these studies presents performers with conditions that are static and largely unchanging (take the standard putting task which is such a popular means of testing motor control in laboratory studies; see Christensen et al., 2015, for a detailed critique). As a result, the tasks are hampered by a lack of real-world complexity - key features that one would find in a naturalistic setting (e.g., changes in environmental conditions or high levels of performance pressure). It is perhaps unsurprising that 'experts' in Beilock's study appeared amnesiac when asked to report what they had focused on when 
performing the putting task. Once a feel for the pace of the putt had been established, motor execution could be initiated and run to completion without any need for the participant to supervise elements of performance or to alter any movement parameters (e.g., timing, force etc).

In summary, task difficulty is likely to have a significant influence on the type of processing engaged in by the skilled performer. Simple laboratory tasks might reveal how automated processes underpin skilled performance when conditions are static and predictable but they do not shed light on the forms of control that might be required when experts face "challenging-but-normal conditions" (Christensen et al., 2016). Additionally, there will often be proponents or properties of a task which have not been automated and this leaves the performer with little choice but to maintain control over task execution (Carr, 2015). This would suggest that automated and controlled processes are actually tightly integrated rather than acting independently of each other. In one of the few empirical studies to explore the latter possibility, Bebko et al. (2005) examined the evolution of controlled and automatic processing in a three-ball bounce juggling task. This task required participants to bounce a ball on the floor alternately from one hand to the other in a V-shape (at least one of the three balls was always in motion). A control task introduced two variations to the juggling task that involved changing the ball weights, sizes and textures in one task whilst using an inclined surface or a flat floor in the other. The basic parameters of the tasks remained the same (i.e., bounce juggling). Control over these adaptations emerged along with the development of automatized skills on the main task. On the basis of these findings, Bebko et al. (2005) presented a model in which controlled and automatic processes emerge orthogonally. According to the authors, "control processes refer to processes that enable adaptation within cognitive schema or motor programs, rendering the programs flexible so that one can respond to changing external or internal demands while still executing the same behaviour" (Bebko et al. 2005, p. 474).

\section{Control and Skilled Action}

In the following sections, we detail various forms of control that experts might utilise during performance. We argue that automated and controlled processes must operate synergistically if the performer is to continue to develop their embodied capacities. In doing so, we will delineate the different types of control skilled agents might employ in seeking to maintain performance proficiency in the face of the huge variety of challenges they face over their lifespan. We will focus on the interaction between automated and controlled processes in two particular contexts (1) when performers seek to update and improve motor execution and (2) when they attempt to stabilise performance proficiency under pressurised conditions. We use Bebko et al.'s (2005) model which focuses largely on the emergence of controlled and automatic processes as performers learn to master motor tasks - to show how the maintenance of skilled or expert action is dependent upon these processes operating in an orthogonal fashion (see Fig. 1). That is, rather than existing on a linear and single continuum, automaticity exists on one continuum (ranging from nonautomatic to automatic processing) and control refers to a separate, orthogonal continuum (ranging from low to highly controlled processing). Using this model, we show how certain performance states are 
characterised by these processes emerging together whilst others are characterised by high levels of automaticity and low levels of control, and vice versa (i.e., one process developing more quickly than the other).

Given the importance we attribute to control processes perhaps we should devote a little more attention to explaining what is meant by the term. In this regard, Fujita et al. (2014) argued that this term has two different meanings. On the one hand, it is defined by the absence of the features (unintentional, unconscious, effortless) that characterise automaticity (bearing in mind that not all automatic processes will be equally characterised by each of these features all of the time, see Fridland, 2014). In other words, controlled processes are simply ones that are not automatic. On the other hand, "controlled" can be defined in terms of its capacity to foster conscious goal-pursuit. Thus, according to Moors (2016), a process is controlled when "a person has a goal ... and there is a causal relation between the goal and the occurence of the desired state" (p. 265). Such conceptualisations seem to cover higher-level goals or intentional states (to do with planning, forethought etc) but do not account for evidence that performers often seek to deliberately control motor execution (e.g., when they experience performance pressure or when they attempt to alter "attenuated" movement patterns) in an effort to maintain performance proficiency. Indeed, there is a wide range of theoretical perspectives and empirical evidence demonstrating the important role cognitive control plays not only in directing strategic aspects of performance but in influencing motor execution also (see Christensen et al. 2016).

Drawing on some of this evidence, philosophers of skilled action have attempted to present a fine-grained analysis of the types of control agents might use during the performance of complex actions (see Fridland, 2014; Mylopoulos \& Pacherie, 2017; Shepherd, 2014 for examples). To illustrate, Christensen et al. (2016) distinguished between three different types of control. First, they argue that strategic control involves formulating the overall game plan one hopes to employ during a temporally extended event (e.g., tennis match). Based on a careful evaluation of one's technical and tactical strengths, and those of the opponent, a tennis player might start a match with the intention of playing largely from the back of the court. However, as the match unfolds,

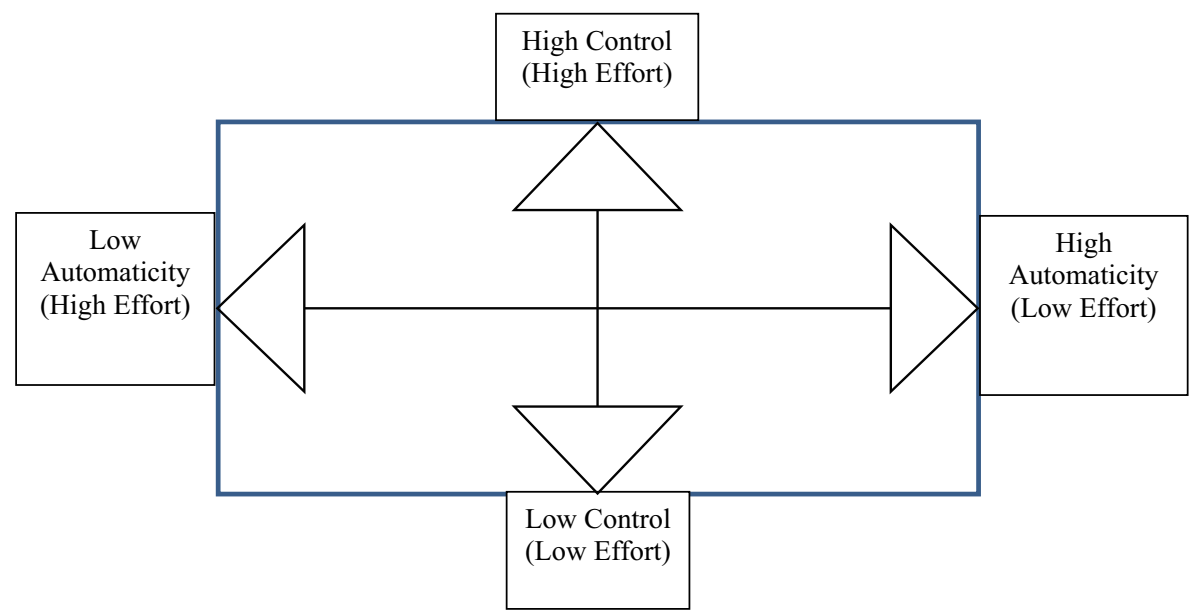

Fig. 1 Orthogonal model of automatic and controlled processing in skilled action (adapted from Bebko et al. 2005) 
the player might realise that this tactic is being neutralised by an opponent who is adopting a similar strategy. To address this, the player might employ situational control whereby he or she determines what actions are suitable for the specific situational challenge they face (if an opponent is wedded to the baseline then one may wish to exploit this by attacking the net). Finally, implementation control involves overseeing the execution of the actions specified by situation control. If one has decided that the most effective strategy is to increase the frequency with which one approaches the net then implementation control will involve automatic adjustments to parametric details of action such as the force and direction of one's volleys (in response, of course, to the opponent's movement).

Not only does received wisdom hold that automaticity underpins the fluent execution of complex skills but, in the psychological literature, control is often presented as the enemy of embodied coping. A large volume of evidence has shown how skilled action is disrupted when experts reinvest conscious attention in proceduralised skills (e.g., Gray, 2004; Jackson et al. 2006). To explain these findings, self-focus models (e.g., Masters', 1992, conscious processing hypothesis and Beilock and Carr's, 2001, explicit monitoring hypothesis) have proposed that anxiety increases athletes' level of self-consciousness and causes them to turn attention away from goal-directed action and inwards towards movement mechanics. As noted above, this shift to self-focused attention is held to prompt a form of "paralysis-by-analysis" whereby athletes attempt to gain control over automated skills. Here, proceduralised control structures that normally operate without interruptions are broken down (or de-chunked) into a sequence of smaller, independent movements, in a manner representative of performance during novice learning. If performers seek to control motor execution by drawing upon explicit rules to manipulate the mechanics of their action during on-line skill execution then one would expect skilled action to be disrupted - especially if the performer had little previous experience focusing on these aspects of technique. That said, we shouldn't take this as evidence that all forms of control will inevitably prove deleterious to performance proficiency.

\section{Bodily "Crises"}

In what follows, we seek to address this issue by discussing a number of situations (i.e., "crises", continuous improvement and performance pressure) that are likely to require flexible control of automated procedures. Let us start by considering how the emergence of certain "crises" might compel the performer to employ controlled modes of processing. The challenges faced by embodied agents who are required to execute complex actions with extraordinary precision over many years means that conscious reflection is a routine feature of their everyday training regimes. They must retain an awareness of their corporeal schema to ensure that everything continues to run smoothly and they have little choice but to consciously intervene - especially when they encounter a variety of what might be termed bodily 'crises'. According to Schilling (2008) crises occur when 'there develops a significant mismatch or conflict between the social and physical surroundings in which individuals live and their biological and bodily potentialities' (p. 16). We argue that these crises represent a relatively ubiquitous feature of the performance environment and present a threat to the athlete's routinised 
ways of acting. Examples include injury, performance slumps and the senescence that confronts the aging body. These events leave the skilled performer with little choice but to consciously override habituated modes of action.

To do so, performers will often seek to address 'attenuated' movement patterns by reinvesting conscious attention in the training context (see Toner et al. 2020). To regain prior levels of performance, athletes in sports such as swimming and javelin throwing have employed a variety of conscious processing strategies to restore or refine habitual movements (Carson et al., 2014; Hanin et al., 2004). Successful cases of technical change have involved performers becoming more consciously aware of the kinaesthetic differences between current (problematic) and desired actions. This is a process that often requires athletes to break down or parse proceduralised movement patterns rather than continuing to practise in a manner which further automates or establishes them. This is undoubtedly a highly effortful process where the performer seeks deliberately and consciously to de-automate habitual movements - often focusing on explicit rules and procedures that guide the execution of the movement. This state is characterized by processing which is slow, attentionally demanding and effortful. Here, habitual actions are broken down into separate units and this may involve freezing various degrees of freedom in an effort to perform the desired movement. In this state, motor execution is rigid and inflexible as the performer seeks to re-parameterize action and gain control over movement proficiency. Considerable conscious resources are required until components of the behavior become "chunked" into single units (motor programs, cognitive schemata; Bebko et al. 2005). Performers undertaking this process would be positioned in the lower left quadrant of our model (characterized by high effort, low automaticity and low control; see Fig. 1). In summary, de-automating an undesired movement pattern and re-automating a new movement requires a high degree of effort. As noted above, the performer would have little control over action at this stage movement outcome will be inconsistent and error-strewn until the new movement is established (see Carson et al. 2014).

\section{Continuous Improvement}

Whilst 'crises' will often prompt conscious modes of processing, we argue that skilled performers' are strongly motivated by a desire to master their craft (see Hardy et al. 2017) and this requires them to remain deeply attentive to embodied capacities. We refer to this process as "continuous improvement" and suggest that it is characterized by a desire to refine and update one's repertoire of skills (Toner and Moran, 2014). There is a tendency in the motor control literature to conflate performance on simplistic laboratory tasks (which, once automatized, require little subsequent oversight) with those requiring considerably more degrees of freedom (e.g., the golf swing) and which are particularly susceptible to bodily crises. It is perfectly understandable why reflection or conscious computation may be unnecessary when one is engaged in mundane activities such as brushing one's teeth or perhaps performing the simplistic sensorimotor skills that are often tested in experimental studies. In these cases, we are likely to have acquired an action which is adequate for the job at hand and have little reason or motivation to consider whether a better or more efficient technique exists. Once acquired and routinized, the action can be initiated and run to completion without the 
need to devote any attention to the mechanics of the movement. Skilled agents, on the other hand, have devoted extraordinary care and attention to the development of their embodied capacities and are heavily invested in the gradual refinement and improvement of their bodily movement (see Montero, 2016).

Indeed, many seem committed to mastering their craft and this appears to be a defining feature of continuous improvement. To illustrate, Hardy et al. (2017) compared the psychosocial biographies of super-elite athletes from Olympic sports (those who had medalled) to those of elite athletes (who had not medalled) and found that the super elite athletes emphasised the importance of both mastery and outcome while the elite athletes focused almost exclusively on winning. The performer who wishes to master his/her craft has little choice but to consciously intervene as excessive proceduralisation is likely to result in 'arrested development' - where performance is constrained and plateaus as a result (Ericsson, 2008). Furthermore, longevity in any skill domain requires participants to keep pace with changes in rules, the introduction of new technology, and advances in understanding as well as dealing with a range of crises. Performers must be able to make intentional modifications to their action in response to these challenges. Avoiding the proceduralisation of skill seems to be an important means by which they achieve this aim (represented by high control and high effort on our model).

\section{Is Optimal Performance Fully Automatic?}

While some researchers might broadly accept that experts can make intentional modifications to their action in the practice context, received wisdom holds that optimal performance in sport is an entirely automatic process (characterised by features such as a lack of awareness, effortless, fast etc). Indeed, the phenomenon known as "flow" is presented as a paradigmatic example of the mindlessness of skilled action (see Dietrich, 2004). This state often accompanies optimal performances and has been characterised by various features such as a merging of action-awareness, a loss of self-consciousness, a sense that one is in total control over one's performance, and the altered sense of time (see Csikszentmihalyi, 1990). Early research on flow presented this state as involving reduced frontal activation (indicative of a reduction in conscious processing) but more recent findings have suggested that supervisory attentional and cognitive control systems of the brain are highly active during flow (see Harris et al., 2017a). For example, Ulrich et al. (2016) discovered that brain areas related to the Multiple Demand (MD) network (which is linked to goal-directed behaviour and selective visual attention) had higher levels of activation during a flow level of difficulty in an arithmetic task than a harder level of difficulty. These findings would suggest that flow states require more attentional effort than previously thought and therefore cannot be considered fully automatic. In a study using a simulated car-racing task designed to promote different levels of flow, Harris et al. (2017b) found that the absorption that accompanies the flow state is based on an efficient but effortful form of attention (as indexed by peak ratings of absorption, peak heart rate and reduced gaze variability).

These neuroscientific and experimental discoveries have been mirrored by recent qualitative evidence which has shown how optimal performance in sport is characterised not by the 'mindlessness' that is typically associated with automated 
processes but, rather, by the flexible deployment of attentional resources in order to maintain performance proficiency under pressurised conditions. In fact, Swann et al. (2017) research on elite athletes' experiences of peak performance indicates that these states are characterised by high levels of automaticity and high levels of control (placing this type of performance state in the upper right quadrant of our model). Interestingly, they have discovered a flow-like state which they termed "letting it happen" and a state which was not characterised by the attentional processes one typically associates with flow or peak performance which they termed "making it happen". Although these states share similar features, "making it happen" was described as a more intense state of optimal arousal with participants experiencing a sense of heightened and effortful concentration. This is intriguing because flow has traditionally been conceptualised as involving an effortless form of attention.

"Letting it happen" seems to be associated with a gradual build-up of confidence over time. One's performance or the fluency of motor execution may improve incrementally over practice sessions until a point where everything 'clicks' into place. As performance improves and one acquires increasing control over performance, the athlete develops the confidence to let motor execution unfold automatically. "Making it happen", on the other hand, is an intentional process involving a conscious decision to increase the attentional resources that one devotes to performance. During this state, task execution continues to unfold in an automatic manner but the performer devotes additional cognitive resources to strategic control. This might happen when the performer realises that they have reached a make-or-break stage of a match/competition (i.e., a situation in which the stakes are particularly high). As a result, the performer must remain sensitive to any changes in the structure of a situation. This, in turn, leads to an increased focus on the strategic aspects of task control (Christensen et al. 2016).

The process of "making it happen" bears conceptual similarity to the phenomenon of "clutch" (which occurs when a participant in a competitive sport succeeds at a point in competition where success or failure has a significant impact on the outcome of the contest; Hibbs, 2010) performance in sport. Both processes are characterised by the use of effortful attention. Evidence in support of this contention can be found in a recent study by Swann et al. (2017) who interviewed 16 athletes from a range of sports about their subjective experiences of clutch performances. The defining characteristics of this state included complete and deliberate focus, intense effort (including trying harder and making a conscious effort), heightened awareness (including awareness of self, self-monitoring), heightened arousal, absence of negative thoughts, and automaticity of skills. Interestingly, the data from this study indicates that both automatic and controlled processes may be involved in clutch states. In stark contrast to earlier accounts of flow/peak performance, Swann et al.'s findings (2017) show how performing at one's best often requires increased effort rather than letting things happen; conscious processing; intensity; and effortful concentration. These athletes induced a clutch state by using self-regulatory processes to increase selfmonitoring and the effort and concentration that they devoted to performance. We argue that flow is characterised by an increase in situational awareness which serves to establish a cognitive and motor configuration appropriate to the context (Christensen et al. 2016).

At first glance, the argument presented above seems to contradict the widely held view that flow states involve effortless (where our attention is held easily) rather than effortful attention (where we must concentrate hard in order to maintain our focus). Interestingly, although Csikszentmihalyi consistently argues that flow is reliant on automated sequences 
of actions he claims that effortless attention is not fully automatic in nature. Thus, Csikszentmihalyi and Nakamua (2010) postulate that 'it is likely that in flow a person is more open, more alert and flexible, within the attentional structure of the activity' (p. 186). These authors argue that skilled actors can filter out extraneous information and focus their attention on the complex and ambiguous stream of information that must be processed and interpreted as the activity unfolds. In this way, they can respond to challenges in an appropriate and context-sensitive manner. It is difficult to envisage how this might happen if the agent is unable to exert control over performance - if they are 'off-task' or fail to keep the intentional goal of the activity activated in consciousness. Flow or optimal experience may very well involve getting caught up in the current of activity but this doesn't mean that performers aren't acutely aware of the quality of their action and the varying problems that may present themselves during the course of skill execution. Retaining situational awareness allows them so respond in a context-sensitive manner by exerting higher-order control over action and this appears to be precisely what happens during clutch performances (high control and high automaticity in our model).

Peak performance states like flow or clutch are characterised by a sense of total control over performance - involving an extremely tight integration between intentional and motor states (Fridland, 2017). Importantly, motor execution here is not of the brute, low-level reflexive variety but is characterised by its variability and flexibility. Fridland (2017) marshals a range of evidence which contradicts the widely held view that automatized basic action routines are ballistic (once initiated they cannot be inhibited) and invariant. If one thinks of automated action in these latter terms then one conceives of movement kinematics as unfolding in a fixed, rigid or predetermined way. We argue that clutch states will only arise if the movement system possesses the flexibility to tailor parametric control to the fine-grained structure of a situation. Skilled agents are capable of this because they possess movement repertoires that are inherently variable (owing to physiological processes and environmental factors) and this allows movement outcomes to be achieved in different ways by dynamical movement systems. Here, movement variability is seen as a key signature of adaptability (Seifert et al. 2013). Variability is believed to provide the movement system with the flexibility required to discover coordination solutions to "achieve the same task goal under dynamically interacting task, environmental and personal constraints" (Chow et al. 2016, p. 37). So, the variability inherent in human movement systems means that automatic basic actions can be adjusted in response to contextually-contingent demands. That said, the athlete can continue to exert higher-order control over the parametric details of action. This performance state is characterised by high speed, high accuracy, flexible control over variations in a task and effortful attention (represented by the top right quadrant of the model; see Fig. 1).

\section{Control and Clutch Performances}

Performance pressure is a ubiquitous feature of elite performers' training and competitive regime. Poor performances can often have important financial and personal implications and this invariably places huge pressure upon athletes. In the next section we detail various forms of control that athletes might employ in seeking to combat the deleterious consequences of performance pressure and to produce clutch performances. A number of studies (see Gucciardi et al., 2010; Hill et al., 2010; Oudejans et al., 2011) 
have identified distraction as the primary mechanism responsible for choking episodes (an acute and considerable decrease in skill execution and performance when selfexpected standards are normally achievable, which is the result of increased anxiety under perceived pressure; Mesagno and Hill, 2013) in skilled performers. Examples of distractions, which have been found to contribute to choking, include possessing unrealistically high expectations and the tendency to use performance outcomes for self-validation. When describing their experiences of choking, athletes in a number of qualitative studies reported focusing heavily on anxiety-related thoughts (e.g., fear of failure/embarrassing outcome) rather than on the process of executing the skill (i.e., task-related information such as focusing on the target). In each of these studies, performers suffered a loss of attentional and emotional control and this affected their ability to direct thoughts to the task at hand. In these cases, distractions operated by influencing "stimulus driven, bottom-up processes over the efficient top-down goaldriven processes" (Derakshan and Eysenck, 2009, p. 170). Focusing on anxiety-related thoughts will disrupt performance not only by impairing attentional inhibition (the process by which, under normal circumstances, people can suppress task-irrelevant cognitive processing and ignore salient yet irrelevant features of a situation) but also by hampering attentional shifting (the process by which people can normally switch their attention in response to changing task requirements).

Various physiological symptoms (e.g., racing heart) typically accompany changes in attentional focus and emotional control. Increases in physiological arousal are likely to prove deleterious to the performance of tasks that require coordination or fine motor control (Collins et al., 2001; Pijpers et al., 2005). According to Hatfield and Kerick (2007) emotional reactivity results in 'noisy output' through the corticospinal tract which results in changes in kinematic, motor unit activity, and autonomic and endocrine functioning. Unfortunately, many performers who experience choking seem to respond by withdrawing effort (representative of low control and low effort on our model). Examples of such avoidance behaviours have been found in experimental studies in which penalty-takers football rush the preparatory and execution stages of performance so that they can get away from the situation as quickly as possible (see Jordet and Hartman, 2008). Other performers might interpret a competitive situation as a threat (owing to a perceived lack of control) and withdraw effort as a form of self-handicapping (a form of proactive selfprotection or excuse-making). In this instance, movement automaticity might remain relatively high but withdrawing effort will only further reduce one's control over performance (exemplified by the bottom right hand quadrant of our model; high automaticity and low levels of cognitive effort might also characterise experts performance in 'easy' or 'normal' conditions). Christensen et al. (2016) speculated that certain forms of negative emotional arousal, such as fear, might interfere with task control by activating an avoidance system that conflicts with sensory, cognitive and motor system processes involved in proficient performance. An alternative possibility is that a focus on taskirrelevant sources of information will reduce the player's situation awareness - leading to attentional slips or resulting in a mis-specified action gist (Christensen et al. 2016; lower right quadrant - that is, high automaticity but low control).

What might the performer do to combat the potentially deleterious effects of performance pressure? In the following section, we marshal a range of empirical evidence which has identified various strategies performers might seek to use in attempting to "make it happen" or to produce clutch performances. Each of these 
strategies represent an attempt to gain control over performance and a move towards the upper right hand quadrant of our model. One possible means of exerting control is through the use of self-talk - a highly popular form of cognitive control used by athletes to alter emotional states or to re-orient their attentional focus during pre-performance routines or skill execution itself. This cognitive strategy involves the use of either covert or overt verbalizations that serve instructional or motivational functions (see review by Van Raalte et al., 2016). This process might include the use of "trigger words" or short, vivid and positively phrased verbal reminders designed to help performers to focus on a specific target or to execute a given action. For example, cues may act as 'triggers' which allow the performer to re-direct one's attention to the task at hand or to prime well-learnt movement patterns (Winter et al., 2014).

If the athlete recognises that they are preoccupied by thoughts unrelated to the immediate task (e.g., the audience, the implications of a poor performance) then cues may re-direct their attention to task-relevant stimuli and allow them to maintain performance efficiency in the face of stressful conditions. Indeed, Christensen et al. posit that the use of 'self-talk' or cue words may serve as a form of 'compensatory' control to alter an emotional or cognitive response - and may represent a means by which the athlete can influence themselves in action. The use of holistic process goals may act as one particularly effective form of compensatory control.

These goals involve a focus on a "single conceptual cue to encapsulate a movement in its entirety" (Mullen et al., 2016, p. 143) and can be used to chunk or trigger larger movement units associated with automatic processes. These cues may be used to reconfigure movement patterns that have become increasingly dysfluent or halting due to high levels of physiological arousal (indicative of a move towards the upper right quadrant of our model). Mullen et al. (2016) compared the efficacy of holistic (e.g., "smooth") and part-process goals (e.g., "outside hand") on driving performance on a race simulator under neutral and pressurised conditions. As predicted by the authors, the use of holistic cue words led to superior performance in the competitive condition. Interestingly, psychophysiological measures (e.g., heart rate variability) revealed that both process goals were associated with an increase in compensatory effort as participants sought to mobilize resources in response to perceived threat (as indicated by higher anxiety scores during the competitive condition). Effort, in this case, involves an increase in higher-level control/ strategic control such as setting new targets or altering one's plan of action.

Holistic cues that are rhythmical in nature may prove useful if stressful conditions result in the degradation of the timing and sequencing of patterned coordination. For example, external rhythmical cues have been shown to reinitiate ambulatory movements in patients suffering from movement disorders. Rhythmical cues may operate by increasing the coupling between the auditory and premotor cortex (see Nombela et al., 2013). Together, the preceding findings suggest that holistic cues might promote increased automaticity of movement control and leave the performer with sufficient attentional resources to engage in strategic control. Here, automated and controlled processes can be found to operate in concert. That said, while this approach will enhance control over motor execution it might reduce the extent to which the performer can respond flexibly to task demands. These cues can be used to re-configure movement patterns but are likely to reduce an athlete's situational awareness - that is, it might compromise their sensitivity to the parametric structure of the performance situation and this, in turn, will influence implementation control. 
These cues might also function as 'core action components' (i.e., actions that when executed in a less optimal manner prove deleterious to performance; see Bortoli et al., 2012) According to Bertollo et al.'s Multi-Action-Plan (MAP) intervention model, focusing on these components of action represent a form of controlled processing which can stabilize or even enhance performance proficiency under pressurized conditions. In a series of recent studies, Bertollo and colleagues have sought to identify the psychophysiological and behavioural mechanisms underlying optimal and suboptimal performance states amongst high-skilled athletes. These authors differentiate between optimal-automated (Type 1) performances and optimal-controlled (Type 2) performances. Their model proposes that athletes can perform effectively in an automatic or flow-state but also when they intentionally exert effort to cope with stressful situations. Type 2 controlled processing is reliant on working memory capacity to deal with novel situations/problems or to address unexpected events. Bortoli et al. (2012) found that a focus on certain core action components including 'body stability' and 'grip and trigger' helped elite shooters to effectively cope with the dysfunctional effects of stress. Bortoli et al. (2012) argue that it will take a minimal amount of conscious control to 'supervise' these components when optimal performance conditions occur but that performers will need to devote increased attention when they encounter challenging situations such as those involving stress or fatigue.

\section{Conclusion}

As noted at the beginning of this paper, linear or serial models of skill learning are unable to explain how skilled performers manage to maintain close control over automated processes. According to these models, highly automated processes are associated with low control and low automaticity is characterised by high control. Drawing heavily on Bebko et al.'s and Christensen et al.'s work we have shown how these processes can operate synergistically. While traditional accounts of skilled performance suggest that action unfolds in an automatic manner, we have argued that skilled action continues to be mediated by a range of control processes. At this point, it is worth briefly considering the higher-order processes that might allow skilled performers to identify appropriate modes of control. We see metacognitive control as a particularly important feature of psychological flexibility or higher order control. Metacognition involves one's knowledge or cognitions about cognitive phenomena (Flavell, 1979). Its primary responsibility is the activation of strategies (i.e., thoughts) to achieve conscious goals (Brick et al., 2016). Brick et al. (2015) recently proposed a metacognitive model comprising two distinct processes: metacognitive skills (e.g., planning, monitoring) and metacognitive experiences (e.g., feelings, judgements and estimates). Drawing on interviews with elite endurance runners, Brick et al. (2015) found that these athletes monitored internal sensory (e.g., perceived exertion) and environmental (e.g., split times) sources of information to generate implicit or explicit metacognitive feelings (e.g., difficulty of the task). Metacognitive monitoring will also involve an awareness about bodily states (e.g., comparing the actual and predicted sensory consequences of an action; see Carruthers, 2015). Conscious control is employed when these feelings make the performer aware of some change in the demands of the task (e.g., pace is too hard, movement is becoming dysfluent). In an 
attempt to exert control, the athlete can utilize a cognitive strategy to address these changes in situational demands (e.g., focus on certain cues) or adapt their pacing strategy. Once initiated, the athlete may remain metacognitively aware of the appropriateness of the adopted focus of attention (Brick et al. 2016).

To conclude, the current paper presents a model of skilled action which explains how controlled and automated processes must be tightly integrated if experts are to maintain performance proficiency. We have marshalled a range of evidence which demonstrates how these processes operate in an orthogonal fashion - as motor execution becomes automated, skilled agents learn to control their movement in increasingly sophisticated ways. Control, on occasion, can take the form of deliberate and intentional efforts to alter or monitor some feature of action but it can also take on a more strategic guise. The development of higher-order control allows the performer to draw on a rich history of conceptual knowledge and memories to inform what type of focus they should adopt during performance. Indeed, the ability to flexibly deploy one's attentional resources is a key determinant of skill development. We have shown how some forms of control enable performers to re-direct their attentional focus and to maintain performance proficiency under pressurised conditions. Whilst much of what the expert does is automated, involving the reliance on bottom-up processes, the unpredictably of performance, alongside the various challenges faced by the embodied agent, means that they must be able to make intentional modifications to action or to influence parametric control during on-line skill execution. As noted, strategic control can be used to alter the course of one's plans - to respond to particularly challenging environmental conditions by increasing the effort one devotes to a task. But the expert can also manipulate the fine-grained structure of action in response to environmental or task perturbations. Such flexibility is required because performers must be able to respond to contextually-contingent demands or to the various bodily 'crises' that they are confronted by over the course of their careers. Skilled action should therefore be seen as entailing the close integration of both automated and controlled processes.

Acknowledgements I note with great sadness that since the submission of this manuscript Aidan Moran passed away on March 16, 2020.

Open Access This article is licensed under a Creative Commons Attribution 4.0 International License, which permits use, sharing, adaptation, distribution and reproduction in any medium or format, as long as you give appropriate credit to the original author(s) and the source, provide a link to the Creative Commons licence, and indicate if changes were made. The images or other third party material in this article are included in the article's Creative Commons licence, unless indicated otherwise in a credit line to the material. If material is not included in the article's Creative Commons licence and your intended use is not permitted by statutory regulation or exceeds the permitted use, you will need to obtain permission directly from the copyright holder. To view a copy of this licence, visit http://creativecommons.org/licenses/by/4.0/.

\section{References}

Anderson, J.R. 1982. Acquisition of cognitive skill. Psychological Review 89: 369-406.

Bebko, J.M., J.L. Demark, N. Im-Bolter, and A. MacKewn. 2005. Transfer, control, and automatic processing in a complex motor task: An examination of bounce juggling. Journal of Motor Behavior 37: 465-474.

Beilock, S.L., and T.H. Carr. 2001. On the fragility of skilled performance: What governs choking under pressure? Journal of Experimental Psychology: General 130: 701-725. 
Beilock, S.L., T.H. Carr, C. MacMahon, and J.L. Starkes. 2002. When paying attention becomes counterproductive: Impact of divided versus skill-focused attention on novice and experienced performance of sensorimotor skills. Journal of Experimental Psychology: Applied 8: 6-16. https://doi.org/10.1037/1076898x.8.1.6.

Beilock, S. 2010. Choke: What the secrets of the brain reveal about getting it right when you have to. London: Constable.

Bortoli, L., M. Bertollo, Y. Hanin, and C. Robazza. 2012. Striving for excellence: A multi-action plan intervention model for shooters. Psychology of Sport and Exercise 13: 693-701. https://doi.org/10.1016 j.psychsport.2012.04.006.

Brick, N., T. MacIntyre, and M. Campbell. 2015. Metacognitive processes in the self-regulation of performance in elite endurance runners. Psychology of Sport and Exercise 19: 1-9.

Brick, N.E., T.E. MacIntyre, and M.J. Campbell. 2016. Thinking and action: A cognitive perspective on selfregulation during endurance performance. Frontiers in Physiology 7: 159.

Carr, T.H. 2015. Strengths and weaknesses of reflection as a guide to action: Pressure assails performance in multiple ways. Phenomenology and the Cognitive Sciences 14: 227-252.

Carruthers, G. 2015. A metacognitive model of the feeling of agency over bodily actions. Psychology of Consciousness: Theory, Research, and Practice 2: 210-221.

Carson, H.J., D. Collins, and B. Jones. 2014. A case study of technical change and rehabilitation: Intervention design and interdisciplinary team interaction. International Journal of Sport Psychology 45: 57-78. https://doi.org/10.7352/IJSP2014.45.057.

Collins, D., B. Jones, M. Fairweather, S. Doolan, and N. Priestley. 2001. Examining anxiety associated changes in movement patterns. International Journal of Sport Psychology 32: 223-242.

Chow, J.Y., K. Davids, C. Button, and I. Renshaw. 2016. Nonlinear pedagogy in skill acquisition: An introduction. London: Routledge.

Christensen, W., J. Sutton, and D.J.F. McIlwain. 2015. Putting pressure on theories of choking: Towards an expanded perspective on breakdown in skilled performance. Phenomenology and the Cognitive Sciences 14: 253-293.

Christensen, W., J. Sutton, and D.J.F. McIlwain. 2016. Cognition in skilled action: Meshed control and the varieties of skill experience. Mind \& Language 31: 37-66.

Csikszentmihalyi, M. 1990. Flow: The psychology of optimal experience. New York: Harper \& Row.

Csikszentmihalyi, M., and J. Nakamura. 2010. Effortless attention in everyday life: A systematic phenomenology. In Effortless attention: A new perspective in the cognitive science of attention and action, ed. $\mathrm{B}$. Bruya, 179-190. Cambridge: The MIT Press.

Derakshan, N., and M.W. Eysenck. 2009. Anxiety, processing efficiency, and cognitive performance: New developments from attentional control theory. European Psychologist 14: 168-176.

Dietrich, A. 2004. Neurocognitive mechanisms underlying the experience of flow. Consciousness and Cognition 13: 746-761.

Ericsson, A.K. 2008. Deliberate practice and acquisition of expert performance: A general overview. Academic Emergency Medicine 15: 988-994.

Evans, J.S.B., and K.E. Stanovich. 2013. Dual-process theories of higher cognition: Advancing the debate. Perspectives on Psychological Science 8: 223-241.

Fitts, P.M., and M.I. Posner. 1967. Human performance. California: Brooks/Cole Publishing Company.

Flavell, J.H. 1979. Metacognition and cognitive monitoring: A new area of cognitive- Developmental inquiry. American Psychologist 34: 906-911.

Fridland, E. 2014. They've lost control: Reflections on skill. Synthese 191: 2729-2750.

Fridland, E. 2017. Skill and motor control: Intelligence all the way down. Philosophical Studies 174: 15391560 .

Fujita, K., Y. Trope, W.A. Cunningham, and N. Liberman. 2014. What is control? A conceptual analysis. In Dual-process theories of the social mind, ed. J.W. Sherman, B. Gawronski, and Y. Trope, 50-68. New York: Guilford.

Furley, P., Schweizer, G., and Bertrams, A. 2015. The two modes of an athlete: dual-process theories in the field of sport. International Review of Sport and Exercise Psychology 8: 106-124.

Gray, R. 2004. Attending to the execution of a complex sensorimotor skill: Expertise differences, choking, and slumps. Journal of Experimental Psychology: Applied 10: 42-54.

Gucciardi, D.F., J.L. Longbottom, B. Jackson, and J.A. Dimmock. 2010. Experienced golfers' perspectives on choking under pressure. Journal of Sport \& Exercise Psychology 32: 61-83.

Hanin, Y., M. Malvela, and M. Hanina. 2004. Rapid correction of start technique in an Olympic-level swimmer: A case study using old way/new way. Journal of Swimming Research 16: 11-17. 
Hardy, L., Barlow, M., Evans, L., Rees, T., Woodman, T., \& Warr, C. (2017). Great British medalists: Psychosocial biographies of super-elite and elite athletes from Olympic sports. In Progress in Brain research (Vol. 232, pp. 1-119). Elsevier.

Harris, D.J., S.J. Vine, and M.R. Wilson. 2017a. Neurocognitive mechanisms of the flow state. Progress in Brain Research 234: 221-243.

Harris, D.J., S.J. Vine, and M.R. Wilson. 2017b. Is flow really effortless? The complex role of effortful attention. Sport, Exercise, and Performance Psychology 6: 103-114.

Hatfield, B.D., and S.E. Kerick. 2007. The psychology of superior sport performance: A cognitive and affective neuroscience perspective. In Handbook of sport psychology, ed. G. Tenenbaum and R.C. Eklund, 3rd ed., 84-109. Hoboken: Wiley.

Hibbs, D. 2010. A conceptual analysis of clutch performances in competitive sports. Journal of the Philosophy of Sport 37: 47-59.

Hill, D.M., S. Hanton, N. Matthews, and S. Fleming. 2010. A qualitative exploration of choking in elite golf. Journal of Clinical Sport Psychology 4: 221-240.

Jackson, R. C., Ashford, K. J., and Norsworthy, G. 2006. Attentional focus, dispositional reinvestment, and skilled motor performance under pressure. Journal of Sport and Exercise Psychology 28: 49-68.

James, W. 1892. Textbook of psychology. London: Macmillan.

Jordet, G., and E. Hartman. 2008. Avoidance motivation and choking under pressure in soccer penalty shootouts. Journal of Sport and Exercise Psychology 30: 452-459.

Masters, R.S. 1992. Knowledge, knerves and know-how: The role of explicit versus implicit knowledge in the breakdown of a complex motor skill under pressure. British Journal of Psychology 83: 343-358.

Masters, R., and J. Maxwell. 2008. The theory of reinvestment. International Review of Sport and Exercise Psychology 1: 160-183.

Mesagno, C., and D.M. Hill. 2013. Definition of choking in sport: Re-conceptualization and debate. International Journal of Sport Psychology 44: 267-277.

Moors, A. 2016. Automaticity: Componential, causal, and mechanistic explanations. Annual Review of Psychology 67: 263-287.

Montero, B.G. 2016. Thought in action: Expertise and the conscious mind. New York: Oxford University Press.

Mullen, R., E.S. Jones, S. Oliver, and L. Hardy. 2016. Anxiety and motor performance: More evidence for the effectiveness of holistic process goals as a solution to the process goal paradox. Psychology of Sport and Exercise 27: 142-149.

Mylopoulos, M., and E. Pacherie. 2017. Intentions and motor representations: The interface challenge. Review of Philosophy and Psychology 8: 317-336.

Nombela, C., L.E. Hughes, A.M. Owen, and J.A. Grahn. 2013. Into the groove: Can rhythm influence Parkinson's disease? Neuroscience \& Biobehavioral Reviews 37: 2564-2570.

Pijpers, J.R., R.R. Oudejans, and F.C. Bakker. 2005. Anxiety-induced changes in movement behaviour during the execution of a complex whole-body task. The Quarterly Journal of Experimental Psychology Section A 58: 421-445.

Oudejans, R.R., W. Kuijpers, C.C. Kooijman, and F.C. Bakker. 2011. Thoughts and attention of athletes under pressure: Skill-focus or performance worries? Anxiety, Stress, \& Coping 24: 59-73.

Shepherd, J. 2014. The contours of control. Philosophical Studies 170: 395-411.

Shilling, C. 2008. Changing bodies: Habit, crisis and creativity. London: Sage.

Shiffrin, R.M., and W. Schneider. 1977. Controlled and automatic human information processing: II. Perceptual learning, automatic attending and a general theory. Psychological Review 84: 127-190.

Seifert, L., C. Button, and K. Davids. 2013. Key properties of expert movement systems in sport. Sports Medicine 43: 167-178.

Sternberg, R.J., \& Sternberg, K. (2009). Cognitive Psychology (6 $6^{\text {th }}$ ed.) Belomnt, CA: Wadsworth.

Swann, C., L. Crust, P. Jackman, S.A. Vella, M.S. Allen, and R. Keegan. 2017. States underlying excellent performance in sport: Toward an integrated model of flow and clutch states. Journal of Applied Sport Psychology 29: 375-401.

Toner, J., and Moran, A. 2014. In praise of conscious awareness: a new framework for the investigation of "continuous improvement" in expert athletes. Frontiers in Psychology 5: 769.

Toner, J., Carson, H. J., Collins, D., and Nicholls, A. 2020. The prevalence and influence of psychosocial factors on technical refinement amongst highly-skilled tennis players. International Journal of Sport and Exercise Psychology 18: 201-217.

Ulrich, M., J. Keller, and G. Grön. 2016. Neural signatures of experimentally induced flow experiences identified in a typical fMRI block design with BOLD imaging. Social Cognitive and Affective Neuroscience 11: 496-507. 
Van Raalte, J.L., A. Vincent, and B.W. Brewer. 2016. Self-talk: Review and sport-specific model. Psychology of Sport and Exercise 22: 139-148.

Winter, S., A.C. MacPherson, and D. Collins. 2014. To think, or not to think, that is the question. Sport, Exercise, and Performance Psychology 3: 102-115.

Wulf, G. 2007. Attention and motor skill learning. Champagne: Human Kinetics.

Publisher's Note Springer Nature remains neutral with regard to jurisdictional claims in published maps and institutional affiliations. 